\title{
Inhibition of PARP Activity Attenuated Hepatic Triglyceride Accumulation in Alcoholic Fatty Liver Disease in Mice
}

Shishun Huang ${ }^{1}$, Bing Zhang ${ }^{1}$, Yingli Chen ${ }^{1}$, Huan Liu ${ }^{2}$, Yang Liu ${ }^{1}$, Xin Li ${ }^{1}$, Zhiwei Bao ${ }^{1}$, Zhenyuan Song ${ }^{3}$ and Zhigang Wang ${ }^{1, *}$

${ }^{1}$ College of Medical Laboratory Science and Technology, Harbin Medical University (Daqing), Daqing 163319, Heilongjiang, China; huangshishun2013@163.com (S.H); 15164582299@163.com (B.Z); chenyingli1979@163.com (Y.C); liuyang901027@163.com (Y.L); 15304865689@163.com (X.L); bzw0628@163.com (Z.B)

${ }^{2}$ Fuxin Center Hospital, Fuxin 123000, Jilin, China;18945941803@163.com (H.L)

${ }^{3}$ Department of Kinesiology and Nutrition, University of Illinois at Chicago, Chicago, IL 60612, USA; song2008@uic.edu (Z.S.)

*Correspondence: bio_mdc_wang@aliyun.com (Z.W.); Tel.: +86-459-8153966;

Fax: 011-86-459-8153968

Abstract: The specific role of nicotinamide adenine dinucleotide (NAD) in hepatic
triglyceride (TG) accumulation in alcoholic fatty liver disease (AFLD) were unclear.
Poly ADP ribose polymerase (PARP) is a NAD-consuming enzyme and its specific
role in the pathogenesis of AFLD is still elusive. In current investigation, we found
that chronic alcohol exposure enhanced hepatic PARP expression and activity and
lowered hepatic $\mathrm{NAD}^{+}$level. PARP activity inhibitor PJ34 decreased the intracellular 
TG content in hepatocyte. Moreover, PJ34 suppressed the gene expression of DGAT1 and DGAT2 and elevated the intracellular $\mathrm{NAD}^{+}$level in hepatocyte. These mechanistic observation was validated in alcohol-fed mice injected with PJ34 intraperitoneally. PJ34 injection attenuated hepatic TG accumulation in alcohol-fed mice. Further, the gene expression of hepatic SERBP-1c, DGAT1 and DGAT2 were lowered by PJ34 injection, while the hepatic $\mathrm{NAD}^{+}$level was augmented by PJ34 injection in alcohol-fed mice. At last, the nicotinamide riboside supplementation alleviated hepatic TG accumulation in alcohol-fed mice. These data indicate that applying PARP specific inhibitor PJ34 by intraperitoneal injection attenuated hepatic $\mathrm{NAD}^{+}$depletion and TG accumulation in alcohol-fed mice, which might be a potential candidate for AFLD therapy.

Keywords: alcoholic fatty liver disease; PARP; PJ34; triglyceride

\section{Introduction}

Alcoholic fatty liver disease (AFLD) is one of the most prevalent forms of chronic liver disease worldwide and has a widespread incidence. It may progress to alcoholic liver disease (ALD) which is a major cause of illness and death in the United States [1,2]. The pathogenesis of AFLD is not well established. It was associated with excessive ethanol consumption which causes hepatic lipid accumulation, enhanced hepatic lipogenesis and inhibits the fatty acid oxidation [3]. AFLD can progress to hepatitis, fibrosis, cirrhosis, which may become hepatocelluar carcinoma and liver-related death. But the mechanisms of AFLD are complicated and 
remain incompletely understood.

Chronic alcohol consumption enhanced lipogenesis and decreased fatty acid oxidation which destroyed the balanced of intracellular fatty metabolism. Alcohol exposure induced the generation of reactive oxygen species (ROS) which caused oxidative stress and lipid accumulation in liver [4]. Excessive alcohol consumption reduced the hepatic nicotinamide adenine dinucleotide $\left(\mathrm{NAD}^{+}\right)$level, which disrupted the balance of $\mathrm{NAD}^{+} / \mathrm{NADH}$ radio and the elevation of the lactate/pyruvate ratio [5]. Ethanol metabolism mediated cellular redox status and disturbed several major hepatic lipid metabolism transcriptional regulators, such as sterol regulatory element binding protein 1c (SREBP-1c), lipin-1, AMP-activated kinase (AMPK), Peroxisome proliferator-activated receptor gamma coactivator 1-alpha (PGC-1 $\alpha$ ), and Forkhead box protein $\mathrm{O} 1$ (FOXO1) [6-8].

Poly ADP ribose polymerase (PARP) is an abundant nuclear protein that plays key roles in a variety of cellular processes, including transcriptional regulation and DNA repair and programmed cell death. It detects and initiates an immediate cellular response to single-strand DNA breaks (SSB) by signaling DNA-repairing enzymes to SSB repair. It is composed of four domains: a DNA-binding domain, a caspase-cleaved domain, an auto-modification domain, and a catalytic domain [9]. As one of the $\mathrm{NAD}^{+}$consumers (Sirtuins, PARPs, and CD38), PARP catalyzed the synthesis of polymeric adenosine diphosphate ribose (pADPr) from $\mathrm{NAD}^{+}$to the target proteins, thereby regulating their activity [10]. Excessive hyperactivation of PARP caused the depletion of intercellular $\mathrm{NAD}^{+}$and ATP levels, which led to 
cellular metabolistic disorder and death. Inhibition of PARP activity promoted atherosclerotic plaque regression, attenuated high-fat diet-induced dysfunction of lipid metabolism and diabetes [11-12]. But the specific role of PARP in AFLD progression was not well investigated.

This study aimed to investigate the effect of PARP inhibitor PJ34 on hepatic lipid accumulation induced by chronic alcohol feeding and the underlying mechanism. We found that PJ34 attenuated hepatic $\mathrm{NAD}^{+}$depletion and triglyceride (TG) accumulation in both hepatocyte and alcohol-fed mice. Our results suggest that PARP inhibitor PJ34 could be applied as a potential therapeutic chemical for AFLD by modulating intracellular $\mathrm{NAD}^{+}$levels.

\section{Materials and Methods}

\subsection{Animal and treatment}

Male C57BL/6 mice ( 8 weeks) weighting $20 \pm 0.5$ (SD) g were obtained from the Harbin Medical University Experimental Animal Center. All studies were approved by the animal Ethical Committee of Harbin Medical University, Daqing, China. The mice were housed in conventional conditions, maintained on a $12 \mathrm{~h}$ light/dark cycle, temperature $23 \pm 2{ }^{\circ} \mathrm{C}$, humidity $50 \pm 5 \%$ and fed standard food and water ad libitum at the animal facility for 1 week before experiment began. All mice were fed for 4 weeks with liquid diets. All diets were purchased from Research Diets Inc (TROPHIC Animal Feed High-Tech, China). Pair-fed diet (TP 4030A, PF) contains $18 \%$ protein, $35 \%$ fat, $47 \%$ carbohydrate and alcohol-fed diet (TP 4030C, AF) contains $18 \%$ protein, $35 \%$ fat, $11 \%$ carbohydrate and $36 \%$ alcohol according to 
Lieber-DeCarli [13]. Forty-five male C57BL/6 mice were then randomly divided into six groups and were fed on respective diets for model development. The groups included: (1) PF + physiologic saline (PF, n=6), (2) PF+ PJ34, (n =6), (3) PF+ nicotinamide riboside (NR, Hangzhou LZ Chemical, China) (n=6); (4) AF + physiologic saline (AF, n=9), (5) AF+PJ34 (n =9), (6) AF+NR (n=9). Treatment: PJ34 as a PARP activity specific inhibitor $(10 \mathrm{mg} / \mathrm{kg} /$ day, Selleck Chemicals, USA) was injected intraperitoneally 3 times a week throughout the experiment. The same volume of physiologic saline ((0.1 ml/20g/day i.p.) was injected intraperitoneally 3 times a week as vehicle-treated. NR as a natural $\mathrm{NAD}^{+}$precursor $(10 \mathrm{mg} / \mathrm{kg} /$ day $)$ was supplemented in the liquid diet throughout the experiment. Food consumption was recorded daily. Mice were weighted weekly on the same day. All animal was sacrificed after an overnight fast. At the end of the experiment, plasma, liver samples and epididymal fat pad samples were harvested.

\subsection{Histological analysis}

The liver samples were fixed in $4 \%$ paraformaldehyde for 2 days, transferred to $70 \%$ ethanol, dehydrated through a serial alcohol gradient, the tissues were embedded in paraffin wax block and cut into $6 \mu \mathrm{m}$ thin sections. All sections were dewaxed in xylene, rehydrated through decreasing concentrations of ethanol, and washed in distilled water. Finally, tissue sections were stained with hematoxylin and eosin (H\&E). The fields were randomly selected and were photographed under $\times 400$ magnification. 


\subsection{Circulating biochemical measurement}

The levels of circulating TG, total cholesterol (TC) and alanine transaminase (ALT) were performed with commercially kits (BioSino, China) according to the manufacturer's protocol. We took a certain amount of plasma into working liquid in proportion. The mixture was incubated in $37{ }^{\circ} \mathrm{C}$ water bath for $10 \mathrm{~min}$. Then, SpectraMAX 190 instrument (Molecular Devices, USA) was used to detect the absorbance of TG and TC at wavelength of $505 \mathrm{~nm}$. As for ALT concentration, we use the commercially enzyme-linked immunosorbent assay (ELISA) kit to measure. The plasma was diluted into 5 times, and incubated with relative working liquid for 30 min in $37{ }^{\circ} \mathrm{C}$ incubator. The absorbance was detected by SpectraMAX 190 instrument at wavelength of $450 \mathrm{~nm}$. The concentration of TG, TC and ALT were calculated by related equation.

\subsection{Hepatic TG and TC measurement}

The liver tissues were washed by PBS for 2 times. $800 \mathrm{mg}$ liver tissue was homogenized in $1 \mathrm{ml} 50 \mathrm{mM} \mathrm{NaCl}$. Liver total lipids were extracted in $7 \mathrm{ml}$ heptanes : isopropanol (3:2) mixture at room temperature in shaking stable for $2 \mathrm{~h}$. They were centrifuged at $3,000 \times \mathrm{g}$ for $5 \mathrm{~min}$. The organic layer was collected to a new tube and dried under a stream of air in the hood. Liver TG content was determined by commercially available kit as described previously.

\subsection{Cell Culture and treatment}

HepG2 cells, a human hepatoma cell line, were purchased from the Institute for biological sciences (Shanghai, China). HepG2 cells were cultured in DMEM high 
glucose culture fluid (Gibco Company, USA) containing 10\% (v/v) FBS, supplemented with $2 \mathrm{mM}$ glutamine, $100 \mathrm{IU} / \mathrm{ml}$ penicillin and $100 \mathrm{mg} / \mathrm{ml}$ streptomycin. They were incubated at $37{ }^{\circ} \mathrm{C}$ in a humidified incubator with $5 \% \mathrm{CO}_{2}$ and $95 \%$ air. For pharmacological studies, cells were washed with phosphate-buffered saline (PBS) 3 times and replaced with serum-free medium for serum starvation. After $12 \mathrm{~h}$ culture, they were treated with PARP inhibitor, PJ34 $(1 \mu \mathrm{M})$ in the absence or presence of Oleic Acid (3.3 $\mu \mathrm{M}$, OA, Sigma, USA) for $24 \mathrm{~h}$. The same volume of serum-free medium acted as the control group.

\subsection{Intracellular TG Determination}

After treatment with PJ34 in presence OA for $24 \mathrm{~h}$, cells harvested at the end of the experiment. For intracellular TG measurement, HepG2 cells were dissolved in 0.3 $\mathrm{ml} \mathrm{NaOH}(50 \mathrm{mM})$ solution per well (24 well plate). Hepatic total lipids were extracted in $1 \mathrm{ml}$ heptanes : isopropanol (3:2) mixture at room temperature in shaking stable for $2 \mathrm{~h}$. They were centrifuged at $3,000 \times \mathrm{g}$ for $5 \mathrm{~min}$. The organic layer was collected to a new tube and dried under a stream of air in the hood. Hepatic TG content was determined by commercially available kit as described previously.

\subsection{Hepatic and Intracellular $N A D^{+}$analysis}

Liver tissue samples and intracellular $\mathrm{NAD}^{+}$levels were extracted by the commercially kit (Suzhou Comin Biotechnology, China). Liver tissue samples and HepG2 cells were harvested after treatment. 5 million cells were collected to centrifugal tube and add $1 \mathrm{ml}$ acid extract liquor. For the liver tissues, $0.1 \mathrm{~g}$ tissue was homogenized in $1 \mathrm{ml}$ acid extract liquor. They were used ultrasonic to broken for 1 
min in ice bath, then bath boiling bath for 5 min. After cooling in ice bath and 10000 $\times \mathrm{g}$ centrifuge in $4{ }^{\circ} \mathrm{C}$ for $10 \mathrm{~min}$. Take the supernatant to a new tube and the same volume of alkaline extract liquor was added. $10000 \times \mathrm{g}$ centrifuge in $4{ }^{\circ} \mathrm{C}$ for $10 \mathrm{~min}$. The supernatant was used to test with the manufacturer's protocol. Finally, the absorbance was detected by SpectraMAX 190 instrument at wavelength of $570 \mathrm{~nm}$. The $\mathrm{NAD}^{+}$concentration in each sample was calculated by related equation.

\subsection{Western blot analysis}

Cells were washed three times with PBS and lysed in RIPA buffer (50 mM Tris (pH7.4), $150 \mathrm{mM} \mathrm{NaCl}, 1 \%$ Triton $\mathrm{X}-100,1 \%$ sodium deoxycholate, $0.1 \%$ SDS, Beyotime Biotechnology, China), supplemented with phenylmethanesulfonyl fluoride (PMSF) (Beyotime Biotechnology, China) for $30 \mathrm{~min}$. And the liver tissues were grinded by glass homogenizer which was lysed in RIPA buffer. Whole cell extracts or tissue extracts were collected by centrifugation at $10,000 \times \mathrm{g}$ at $4{ }^{\circ} \mathrm{C}$ for $15 \mathrm{~min}$. Protein concentration was determined using Enhanced BCA Protein Assay Kit (Beyotime Biotechnology, China). Equal protein amounts $(30 \mu \mathrm{g})$ were subjected to $10 \%$ or $8 \%$ SDS-PAGE depending on the molecular weight of desired proteins and transferred to Nitrocellulose Transfer Membrane (Pall Corporation, USA). The membranes were blocked with 5\% powdered milk diluted in Tris-buffered saline (TBS) with $0.05 \%$ Tween 20 (TBST) for 1 hour at room temperature and incubated with primary antibodies at $4{ }^{\circ} \mathrm{C}$ overnight. Primary antibodies used were: mouse monoclonal IgG3 anti-pADPr (10H) (Santa Cruz Biotechnology, USA), rabbit monoclonal anti-PARP (46D11) (Cell Signaling Technology, USA), rabbit polyclonal 
anti-SREBP-1c (Bioss, China), rabbit polyclonal anti-DGAT1 (Bioss, China), rabbit polyclonal anti-DGAT2 antibody (Bioss, China), mouse monoclonal anti- $\beta$-actin (ZSGB-BIO, China). The antigen-primary antibody complexes were washed and then incubated with HRP-conjugated secondary antibodies at room temperature for $1 \mathrm{~h}$. Secondary antibodies used were: goat anti-mouse and anti-rabbit horseradish peroxidase-conjugated (ZSGB-BIO, China). Peroxidase was detected using enhanced chemiluminescence (ECL) Western blotting detection reagents (Haigene, China). Densitometric analysis was performed using Quantity One software (Bio-rad, USA).

\subsection{RNA Extraction and Real-time PCR Assays}

Total RNAs were extracted from liver tissues or HepG2 cells. Total RNAs were isolated using TRlzol Reagent (HaiGene, China) according to the manual. For each sample, $1 \mu \mathrm{g}$ of total RNAs were reverse transcribed using Golden 1st cDNA Synthesis Kit (HaiGene, China) as described by the manufacturer $\left(30{ }^{\circ} \mathrm{C}\right.$ of $15 \mathrm{~min}$; $55{ }^{\circ} \mathrm{C}$ of $50 \mathrm{~min} ; 95{ }^{\circ} \mathrm{C}$ of $10 \mathrm{~min}$ ). Real-time PCR of SREBP-1, diglyceride acyltransferase 1 (DGAT1), DGAT2 and 18s rRNA mRNA was performed in Applied Biosystems 7300 Real-time PCR system (Applied Biosystems, USA) by using the SYBR Green qPCR Mix (Roche, Switzerland) under the following conditions: $95{ }^{\circ} \mathrm{C}$ for $10 \mathrm{~min}, 45$ cycles of $95{ }^{\circ} \mathrm{C}$ for $10 \mathrm{~s}, 58{ }^{\circ} \mathrm{C}$ for $20 \mathrm{~s}$ and $72{ }^{\circ} \mathrm{C}$ for $30 \mathrm{~s}$. To amplify the target genes, all primers were purchased from Genscript Biotechnology (Nanjing, China). Quantitative normalization of the cDNA in each sample was performed using the $18 \mathrm{~s}$ rRNA gene as an internal control. Real-time PCR assays were performed in duplicate for each sample, and the mean value was used for the calculation of mRNA 
expression levels.

\subsection{Statistical analysis}

Statistical analysis was performed using one-way analysis of variance and Newman-keuls test by OriginPro7.5 software (OriginLab company, Austin, USA) and Graph Pad Prism version 5 (Graph Pad Software Inc. San Diego,California). Data were presented as the mean \pm standard error. Values of $\mathrm{P}<0.05$ were considered to indicate a statistically significant difference.

\section{Results}

3.1. Chronic Alcohol Consumption induced hepatic fat accumulation and liver injury in mice

C57BL/6 mice were fed with Lieber-DeCarli alcohol-containing diet for 4 weeks. Liver samples were harvested for histological examination and the hepatic TG content was detected. As shown in our results, chronic alcohol feeding significantly decreased body weight and epididymal fat pad weight/body weight rate (Figure 1A,C) in comparison to PF group, whereas the liver weight/body weight rate was significantly increased (Figure 1B). Chronic alcohol consumption increased hepatic TG content and circulating TG level (Figure 1E,F). Chronic alcohol exposure increased circulating ALT level (Figure 1D) and hepatic TC level (Figure 1G). The severity of AFLD was analyzed by staining with $\mathrm{H} \& \mathrm{E}$ staining (Figure $1 \mathrm{I}, \times 400$ ). In the AF group, the lobular structures was destroyed and widely distributed, with more lipid droplets when compared with the PF group. The data showed that chronic alcohol consumption induced hepatic lipid accumulation and liver injury. 

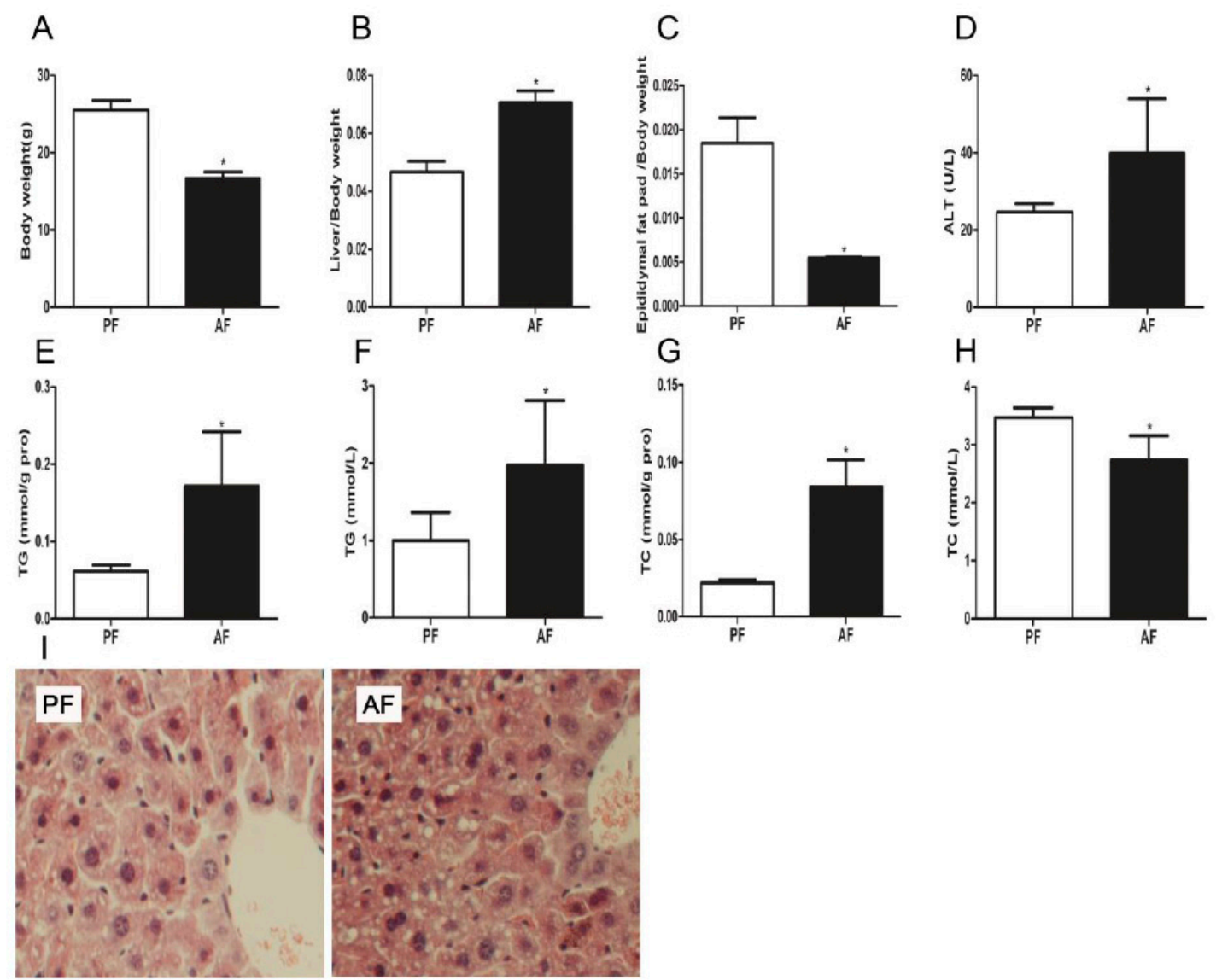

Figure 1. Chronic Alcohol Consumption induced hepatic fat accumulation and liver injury in C57BL/6 mice. Mice were fed with Lieber-DeCarli alcohol-containing diet for 4 weeks. Chronic alcohol feeding lowered body weight (A) and increased Liver/body weight rate (B) in comparison to PF group. Epididymal fat pad /body weight rate (C) also decreased by chronic alcohol feeding. The levels of circulating ALT (D), hepatic TG (E), circulating TG (F) and hepatic TC (G) were significant increase by chronic alcohol consumption. But the circulating TC level $(\mathrm{H})$ was lowered in comparison to PF group. Chronic alcohol feeding enhanced hepatic fat accumulation by staining of liver sections with $\mathrm{H} \& \mathrm{E}(\times 400)(\mathrm{I})$. Values displayed are expressed as the mean $\pm \mathrm{SD}$ ( $\mathrm{n}=6$ per group). Asterisk $(* p<0.05)$ indicates significant differences compared to the pair-fed group. PF, pair-fed; AF, alcohol-fed. 
3.2. Chronic alcohol feeding enhanced PARP expression, $p A D P r$ synthesis and NAD depletion in mice

After feeding with ethanol-containing liquid diet for 4 weeks, liver samples were harvested for further experiment. Total proteins were extracted from liver samples. Western blot was used to detect PARP expression and activation in alcohol-fed mice. Our results showed that chronic alcohol feeding elevated PARP protein expression (Figure 2A,B) and activity, which was showed by higher pADPr conjugated with proteins (Figure 2A,C), in comparison to the PF group. Moreover, chronic alcohol consumption decreased hepatic $\mathrm{NAD}^{+}$level (Figure 2D) and increased the gene expression and protein abundance in TG anabolism, such as SREBP-1c, DGAT1 and DGAT2 (Figure 2E-J) compared with the PF group. Thus, all the results indicate that chronic alcohol consumption-induced hepatic TG accumulation was associated with hepatic PARP overexpression and activation, and the following hepatic $\mathrm{NAD}^{+}$ depletion. 

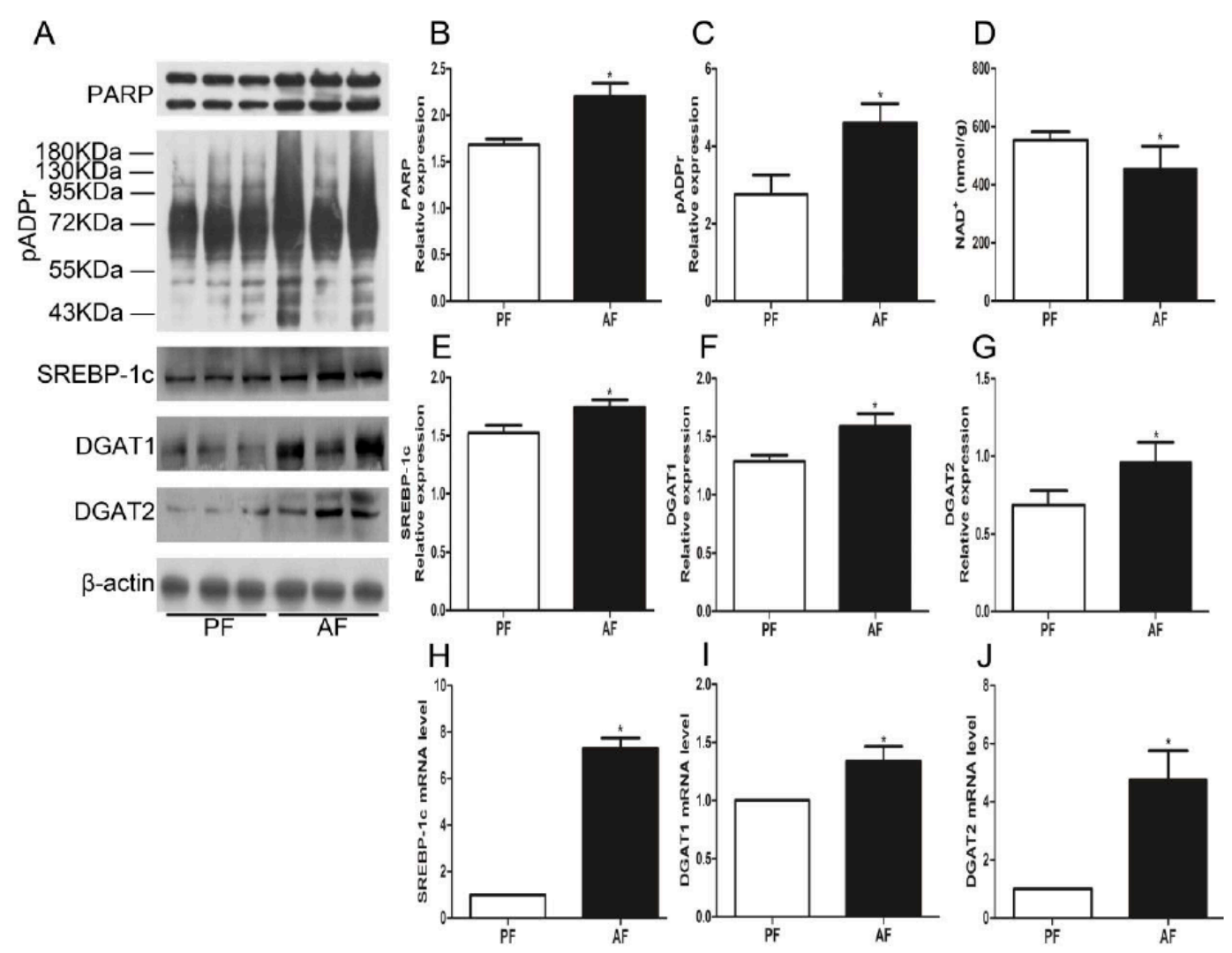

Figure 2. Chronic alcohol feeding enhanced PARP expression, pADPr synthesis and $\mathrm{NAD}^{+}$depletion in alcohol-fed mice. Total proteins were extracted from liver tissues and subjected to Western blot for expression of PARP, pADPr, SREBP-1c, DGAT1 and DGAT2 (A) with specific antibodies. Chronic alcohol exposure increased the expression of hepatic PARP protein (B) which was activity for catalyzing the pADPr formation (C) and lowered hepatic $\mathrm{NAD}^{+}$content in comparison to PF group (D). The densitometric analysis was showed that chronic alcohol feeding increased the expression of SREBP-1c (E), DGAT1 (F) and DGAT2 (G) proteins. Relative genes expression of SREBP-1c (H), DGAT1 (I) and DGAT2 (J) were significant higher by chronic alcohol consumption in comparison to PF group. The data are expressed as the mean $\pm \mathrm{SD}$ ( $\mathrm{n}=6$ per group). Asterisk $(* p<0.05)$ indicates significant differences compared with PF group. 
3.3. Inhibition of PARP activity increased $N A D^{+}$level and lowered intracellular $T G$ content in hepatocyte

The HepG2 cells were used to investigate whether PARP inhibition modulated hepatic lipid accumulation and the underlying mechanism. As shown in our results, PJ34 exposure $(1 \mu \mathrm{M})$ for $24 \mathrm{~h}$ decreased pADPr synthesis (Figure 3C) but had no effect on the protein abundance of PARP (Figure 3A,B) in hepatocyte. Furthermore, we found that intracellular $\mathrm{NAD}^{+}$content was elevated by PJ34 (Figure 3E) while intracellular TG content was lowered by PJ34 treatment (Figure 3E). These results demonstrated that PARP inhibition augmented intracellular $\mathrm{NAD}^{+}$and decreased intracellular TG accumulation in HepG2 cells.
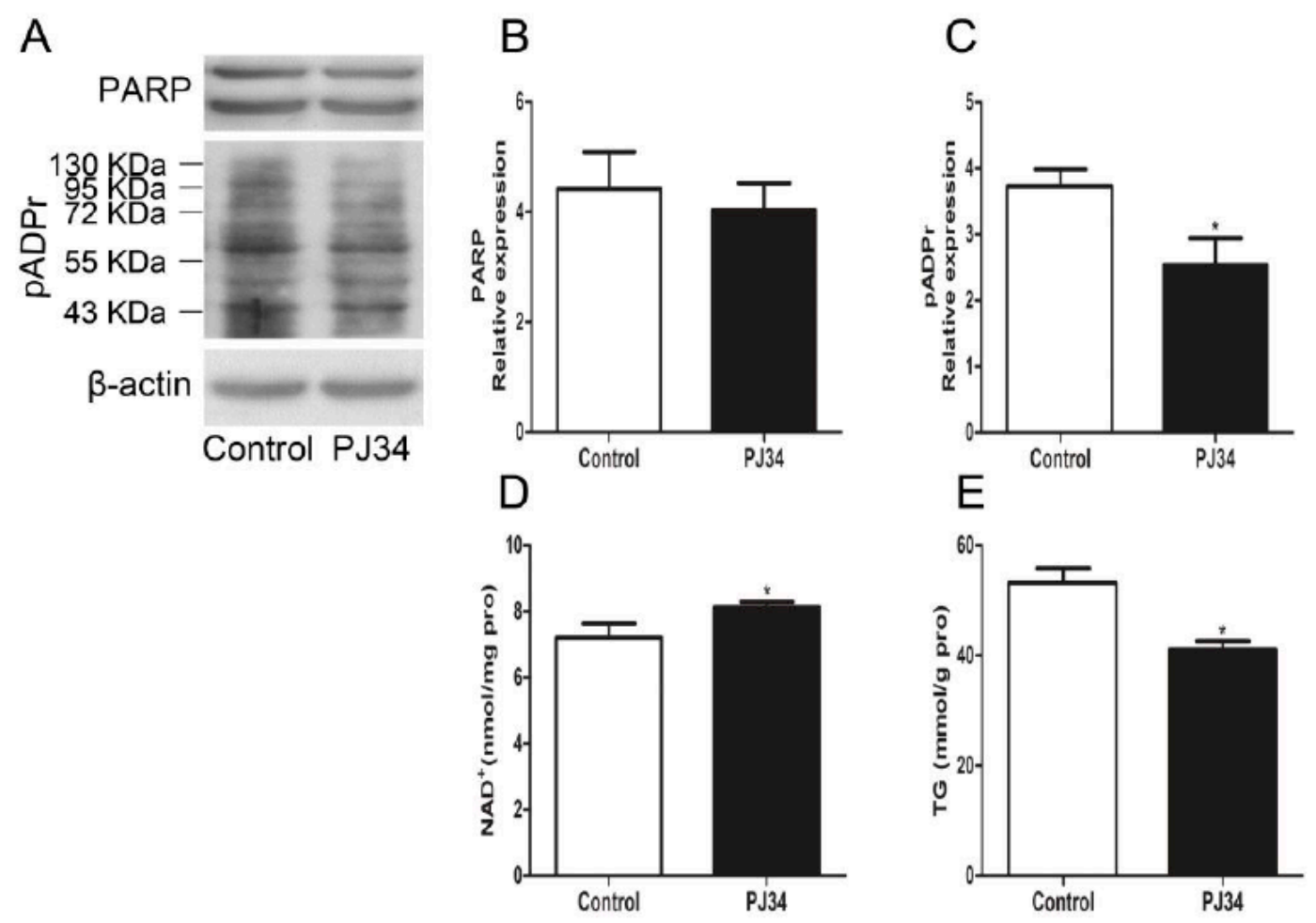

Figure 3. Inhibition of PARP activity augmented $\mathrm{NAD}^{+}$level and decreased intracellular TG content in hepatocyte. HepG2 cells were exposed to complete DMEM medium containing PJ34 $(1 \mu \mathrm{M})$ for $24 \mathrm{~h}$. The expression of intracellular 
PARP and pADPr were detected by Western blot (A). PJ34 treatment had no significant change with the expression of PARP (B) and decreased the synthesis of pADPr (C). PJ34 treatment elevated intracellular NAD ${ }^{+}$content (D). Moreover, PJ34 treatment also decreased intracellular TG content in cells (E). All values were denoted as means \pm SD from three or more independent batches of cells. ${ }^{*} p<0.05$ compared to control group.

3.4. Inhibition of PARP activity suppressed the expression of the genes in TG anabolism

To gain insight into the mechanism underlying PJ34 decreased intracellular TG accumulation, HepG2 cells were exposed to PJ34 $(1 \mu \mathrm{M})$ in complete DMEM medium with OA addition for 24 hours. The critical genes in TG anabolism were detected by RT-PCR. We found that the gene expression of DGAT1 and DGAT2 were significantly lowered by PJ34 treatment (Figure 4B,C) but the gene expression of SREBP-1c changed little (Figure 4A) in HepG2 cells. Consistent with the PJ34-triggered elevation of intracellular TG content (Figure 3E), our results demonstrated that applying PJ34 to inhibit PARP activity decreased TG accumulation, and the underlying mechanism is involved in PJ34-inhibited gene expression of DGAT1 and DGAT2 in TG anabolism in HepG2 cells. 

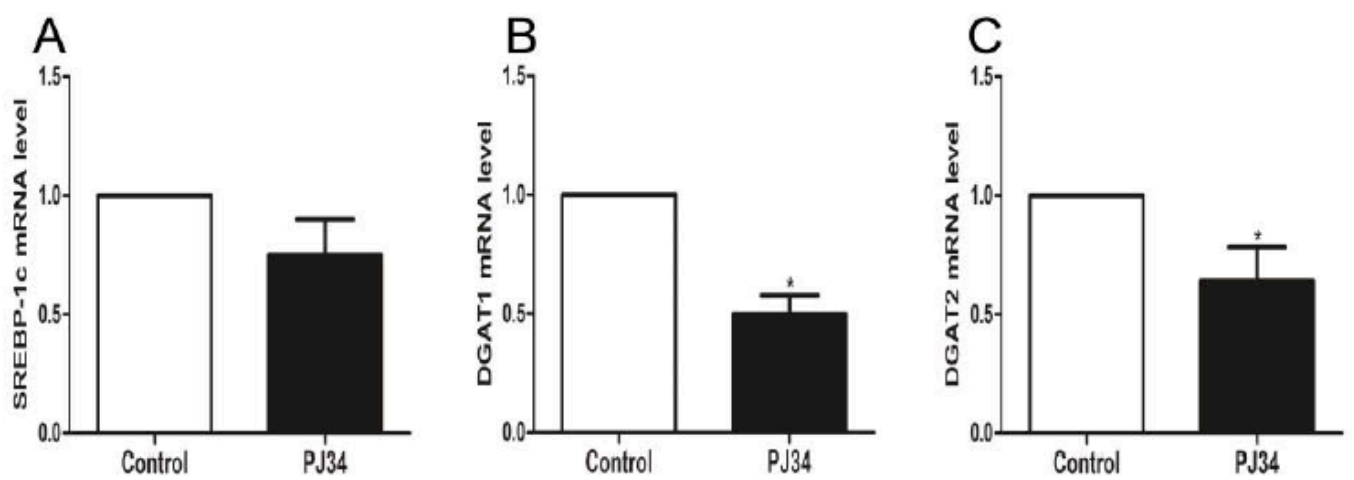

Figure 4. Inhibition of PARP activity suppressed the expression of critical genes in TG anabolism. HepG2 cells were treated with PJ34 $(1 \mu \mathrm{M})$ in present OA for $24 \mathrm{~h}$. Total RNAs were extracted from cells. The mRNA of critical genes was detected by RT-PCR. PJ34 treatment changed a little of intracellular SREBP-1c (A) and lowered intracellular gene expression of DGAT1 (B) and DGAT2 (C). All values were denoted as means \pm SD from three or more independent batches of cells. $* p<0.05$ compared to control group.

\subsection{PJ34 injection attenuated hepatic TG accumulation in alcohol feeding mice}

To detect the specific role of PARP inhibition in the pathogenesis of AFLD, C57BL/6 mice were exposed to alcohol-containing liquid diet with/without PJ34 intraperitoneal injection for 4 weeks. As shown in our results, PJ34 injection increased body weight (Figure 5A) and decreased the liver weight/body weight rate (Figure 5B), but the epididymal fat pad weight/body weight rate had no significant change (Figure 5C) compared with the AF group. Moreover, PJ34 injection decreased hepatic TG content and circulating TG level in comparison to the AF group (Figure 5E,F). In our results, PJ34 injection had no roles in hepatic TC and circulating TC and ALT level in 
comparison to the AF group (Figure 5D,G,H). Additionally, H\&E staining showed that PJ34 injection alleviated hepatic lipid accumulation and the lobular structures were more intact in $\mathrm{AF}+\mathrm{PJ} 34$ group compared with $\mathrm{AF}$ group (Figure $5 \mathrm{I}$ ).
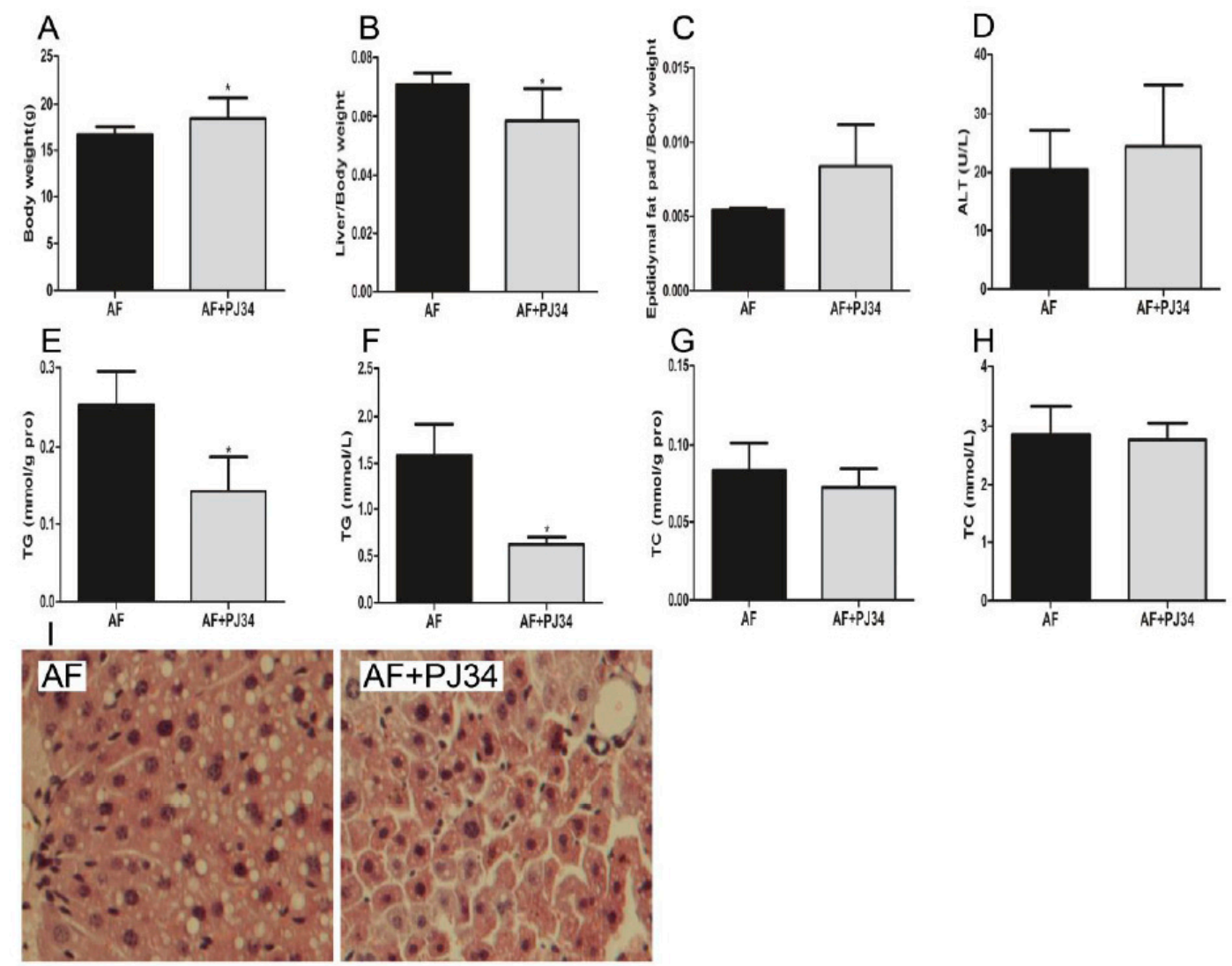

Figure 5. PJ34 injection attenuated hepatic TG accumulation in alcohol-fed mice.

C57BL/6 mice were fed with alcohol-containing liquid diet with/without PJ34 intraperitoneal injection for 4 weeks. In comparison to AF group, PJ34 injection augmented body weight (A) and lowered liver/body weight rate (B). But PJ34 injection had no significant change of epididymis fat/body weight rate (C) and circulating ALT level (D). PJ34 intraperitoneal injection decreased hepatic TG content (E) and circulating TG level (F), but had no difference with hepatic TC content (G) and circulating TC level $(\mathrm{H})$ in comparison to AF group. Liver sections were stained 
with $\mathrm{H} \& \mathrm{E}(\times 400)(\mathrm{I})$. The data are expressed as the mean $\pm \mathrm{SD}(\mathrm{n}=6$ per group); ${ }^{*} p<0.05$ compared to AF group.

\subsection{PJ34 injection elevated hepatic $N A D^{+}$level and lowered genes expression in TG} anabolism in alcohol-fed mice

To detect the underlying mechanism for PJ34-alleviated TG accumulation in the pathogenesis of AFLD, C57BL/6 mice were fed with liquid diets containing alcohol with PJ34 injection for 4 weeks. We found that PJ34 injection suppressed hepatic PARP activation by decreasing pADPr level in comparison to AF group (Figure 6A-C). PJ34 injection alleviated hepatic alcohol-induced $\mathrm{NAD}^{+}$depletion compared with AF group (Figure 6D). Furthermore, the expression of critical genes including SREBP-1c, DGAT1 and DGAT2 were significantly lowered by PJ34 injection in comparison to AF group (Figure 6E-G). All the data demonstrated that PJ34 injection alleviated hepatic TG accumulation via increasing $\mathrm{NAD}^{+}$level and inhibiting TG anabolism in the liver in alcohol-fed mice.
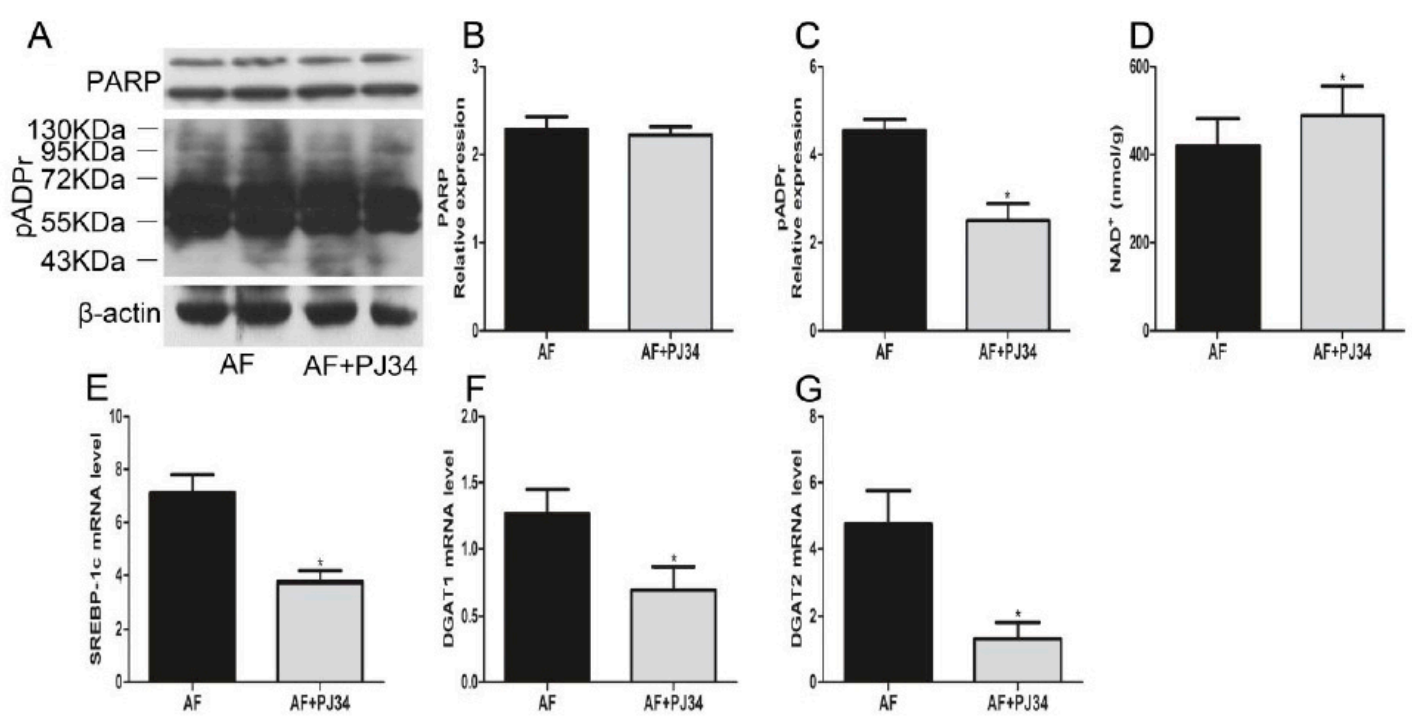
Figure 6. PJ34 injection elevated hepatic $\mathrm{NAD}^{+}$level and lowered gene expression in TG anabolism in alcohol-fed mice. Male C57BL/6 mice were fed with alcohol-containing liquid diets and injected intraperitoneally with/without PJ34 for 4 weeks. Total proteins were extracted from liver tissues and used to detect the expression of PARP and the synthesis of pADPr (A) with specific antibodies. PJ34 injection had no effect with PARP expression (B) and decreased pADPr synthesis (C). PJ34 injection reduced hepatic $\mathrm{NAD}^{+}$depletion in comparison to AF group (D). PJ34 injection significantly alleviated hepatic critical genes expression of SREBP-1 (E), DGAT1 (F) and DGAT2 (G) in comparison to AF group. The data are expressed as the mean $\pm \mathrm{SD}$ ( $\mathrm{n}=6$ per group); ${ }^{\mathrm{p}}<0.05$ compared to the AF group.

\subsection{NR supplementation attenuated hepatic TG accumulation in alcohol feeding mice}

To further demonstrate the specific role of $\mathrm{NAD}^{+}$in $\mathrm{TG}$ anabolism, C57BL/6 mice were fed with alcohol-containing liquid diet supplemented with NR for 4 weeks. We found that NR supplementation increased body weight (Figure 7A) and decreased liver weight/body weight rate (Figure 7B) in comparison to AF group. And no significant change were observed in epididymal fat pad weight/body weight rate (Figure 7C) and hepatic TC content (Figure 7E) in alcohol-fed mice after NR supplementation. Importantly, NR supplementation elevated hepatic $\mathrm{NAD}^{+}$content (Figure 7F) and alleviated hepatic TG accumulation (Figure 7D) in alcohol-fed mice. All the data suggest that elevating hepatic $\mathrm{NAD}^{+}$content by NR supplementation attenuated chronic alcohol consumption-induced hepatic TG accumulation. 

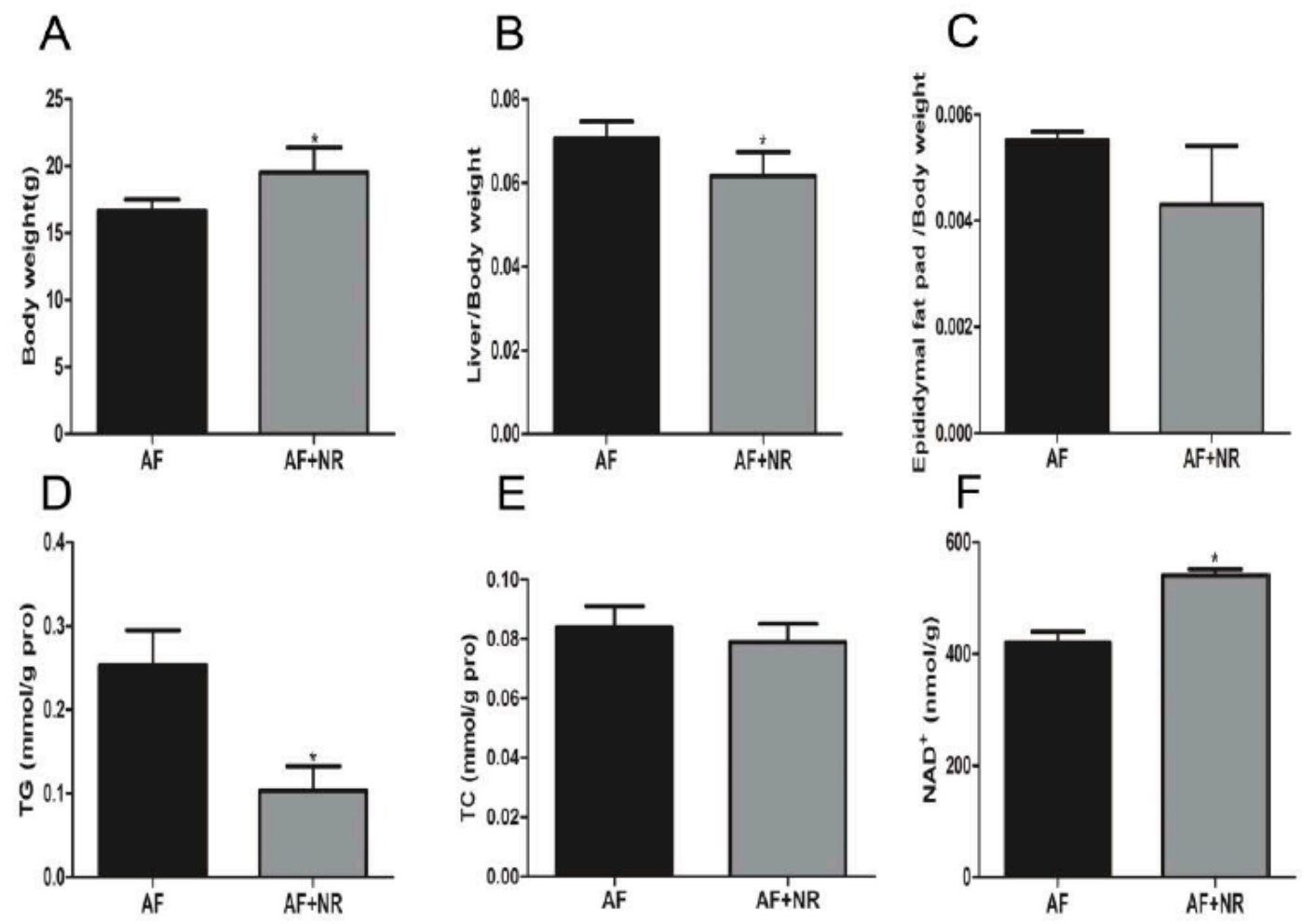

Figure 7. NR supplementation attenuated hepatic TG accumulation in alcohol feeding mice. Mice were fed alcohol-fed liquid diets with NR supplementation for 4 weeks. NR supplementation augmented body weight (A) and decreased liver/body weight rate compared with AF group (B), whereas NR had no change with epididymal fat $\mathrm{pad} /$ body weight rate (C). NR supplementation decreased hepatic TG content (D) and had no change with hepatic TC level (E) in comparison to AF group. Supplement with $\mathrm{NR}$ elevated hepatic $\mathrm{NAD}^{+}$content $(\mathrm{F})$ in $\mathrm{AF}+\mathrm{NR}$ group. Asterisk $(* p<0.05)$ indicates significant differences compared to AF group. 


\section{Discussion}

In the current study, we demonstrated that PARP inhibitor PJ34 alleviated TG accumulation in both hepatocyte and the liver of alcohol-fed mice and the underlying mechanism is involved in the PJ34-elevated $\mathrm{NAD}^{+}$level and PJ34-inhibited gene expression of SREBP-1c, DGAT1 and DGAT2 in the liver in alcohol-fed mice. All the data suggested that PJ34 has a therapeutic potential in AFLD via increasing the hepatic $\mathrm{NAD}^{+}$content and decreasing hypertriglyceridemia and hepatic TG accumulation.

Various elegant publications have been shown that PARP has been gaining recognition as a central regular signaling molecule in numerous diseases, including cancer, energetic metabolism, inflammation, shock, cardiovascular diseases, diabetes and fatty liver disease [14-17]. High-fat high-sucrose (HFHS)-diet increased PARPs activity in mice, showed by the ADP-ribose polymerization of proteins catalyzed by PARPs. NAD ${ }^{+}$was used to produce ADP-ribose for PARPs-catalyzed ADP-ribose polymerization, followed by reduced intracellular $\mathrm{NAD}^{+}$level and disrupted the mitochondrial function and the resulting hepatic lipid accumulation [18]. In line with these investigation, we found that chronic alcohol feeding mice caused PARP over activation and higher levels of ADP-ribose polymers of proteins compared to the $\mathrm{PF}$ group. That suggests PARP activation was involved in the pathogenesis of AFLD. As a $\mathrm{NAD}^{+}$consumer, PARP activation promoted the accumulation of ADP-ribose polymers and $\mathrm{NAD}^{+}$depletion in many diseases [19-22]. $\mathrm{NAD}^{+}$depletion impaired cellular redox capacity and metabolic transformation $[23,24]$. In current investigation, 
chronic alcohol feeding for 4 weeks caused the decline of hepatic $\mathrm{NAD}^{+}$level, resulting from hepatic PARP activation. It was reported that $\mathrm{NAD}^{+}$overconsumption leaded to the metabolic disorder of fatty acids and the following hepatic lipid accumulation [24,25]. Increasing evidence showed that the alteration of PARP expression and activity was associated with the lipid metabolism [26-28]. Therefore, all the data and publication indicate that chronic alcohol consumption-induced the activation of hepatic PARP and the decline of hepatic $\mathrm{NAD}^{+}$content have critical roles in hepatic TG accumulation. And the pharmacological modulation of hepatic PARP activity might be a potential candidate for AFLD therapy.

The similar consideration was seen in several current reports. PARP inhibitor olaparib improved hepatic fatty acid oxidation and NAFLD induced by HFHS-diet [18]. Pharmacological inhibition of PARP or genetic deletion of PARP1 can restored the hepatic $\mathrm{NAD}^{+}$content and increased SIRT1 activation, which decreased hepatic TG accumulation in both alcoholic and nonalcoholic steatohepatitis (ASH/NASH) [25]. In current investigation, we found that PJ34 injection for 4 weeks lowered both circulating TG and hepatic TG level in alcohol-fed mice, but no significant change was observed for circulating and hepatic TC level in the same conditions. The mechanistic investigations in HepG2 cells showed that PJ34 significantly decreased the intracellular TG level as well. But the expression level of PARP had little change in PJ34-treated cells. Further, PJ34 reduced the gene expression of DGAT1 and DGAT2, both of which were key enzymes in TG anabolism. But there was no significant change in the gene expression of SREBP-1c, the lipogenic transcriptional 
factor in PJ34-treated hepatocyte in comparison to the control group. And whether the transcriptional activity of SREBP-1c was lowered by PJ34 exposure needed to be confirmed in further investigation. All the mechanistic observation in hepatocyte was validated in alcohol-fed mice. PJ34 injection for 4 weeks decreased hepatic gene expression of SREBP-1c, DGAT1 and DGAT2 in alcohol-fed mice. Overall, our results suggested that PARP inhibitor PJ34 attenuated hepatic TG accumulation in AFLD progression and the underlying mechanism is involved in PJ34-suppressed gene expression of DGAT1 and DGAT2 in TG anabolism.

Since hepatic PARP-related $\mathrm{NAD}^{+}$alteration was associated with the dysfunction of TG accumulation in the progression of non-alcoholic steatohepatitis [18,25], whether it accounted for the progression of alcoholic steatohepatitis needed to be demonstrated. In our study, chronic alcohol feeding significantly decreased hepatic $\mathrm{NAD}^{+}$level in comparison to the PF group. And PARP inhibitor PJ34 injection elevated hepatic $\mathrm{NAD}^{+}$level in alcohol-fed mice. The data in HepG2 cell lines showed that PJ34 elevated intracellular $\mathrm{NAD}^{+}$content as well. All the change of hepatic $\mathrm{NAD}^{+}$by PARP inhibition is associated hepatic TG alteration. To further demonstrate PARP-regulated $\mathrm{NAD}^{+}$in alcoholic hepatic TG metabolism, we supplemented NR in alcohol-diet for 4 weeks at the same time. Recent study showed that $\mathrm{NR}$ as a $\mathrm{NAD}^{+}$precursor increased $\mathrm{NAD}^{+}$in both skeletal muscle and liver which enhanced oxidation metabolism and protected against high-fat diet-induced metabolic dysfunction via activated sirtuin1 and sirtuin3 [29]. In our study, we found that NR supplementation increased the body weight and lowered the liver weight in 
alcohol-fed mice. NR supplementation elevated hepatic $\mathrm{NAD}^{+}$content and attenuated hepatic TG accumulation in alcohol-fed mice. All the data suggest that high level of $\mathrm{NAD}^{+}$supplementation alleviated alcoholic hepatic $\mathrm{NAD}^{+}$depletion and $\mathrm{TG}$ accumulation in the progression of AFLD. PARP activation-induced low level of hepatic $\mathrm{NAD}^{+}$might be one reason accounting for the pathogenesis of AFLD.

In general, our investigation provided the primary evidence that exogenous PARP pharmacological inhibitor PJ34 was effective against alcohol-induced hepatic TG accumulation through enhancing hepatic $\mathrm{NAD}^{+}$content and suppressing the gene expression of DGAT1 and DGAT2 in the liver in alcohol-fed mice. Chemical inhibitor PJ34 has the therapeutic potential in AFLD progression via restoring hepatic NAD ${ }^{+}$ depletion and lowering hepatic TG anabolism.

Acknowledgments: This work was supported by National Natural Science Foundation (81370523), Postdoctoral Science Foundation Special Project (201104420), China Postdoctoral Science Foundation General Project (20100471022), Heilongjiang Young Key Academic Staff support program (1251G039), Science and Technology Bureau of Daqing (szd-2015-02) and Postgraduate Innovative Program (YJSCX2015-59HYD).

Author Contributions: Z.W., Z.S. and S.H. conceived and designed the studies; Z.W. and Z.S. coordinated the study; S.H., Z.B., Y.C., H.L., Y.L. and X.L. performed the experiments; Y.C. Y.L. and B.Z. contributed to the data collection and analyzed the data; S.H. and Z.W. wrote the original manuscript.

Conflicts of Interest: The authors declare no conflict of interest. 


\section{References}

1. Purohit, V.; Russo, D.; Coates, P. M., Role of fatty liver, dietary fatty acid supplements, and obesity in the progression of alcoholic liver disease: introduction and summary of the symposium. Alcohol 2004, 34 (1), 3-8.

2. Livero, F. A.; Acco, A., Molecular basis of alcoholic fatty liver disease: From incidence to treatment. Hepatology research : the official journal of the Japan Society of Hepatology 2016, 46 (1), 111-23.

3. Rogers, C. Q.; Ajmo, J. M.; You, M., Adiponectin and alcoholic fatty liver disease. IUBMB life 2008, 60 (12), 790-7.

4. Roskams, T.; Yang, S. Q.; Koteish, A.; Durnez, A.; DeVos, R.; Huang, X.; Achten, R.; Verslype, C.; Diehl, A. M., Oxidative stress and oval cell accumulation in mice and humans with alcoholic and nonalcoholic fatty liver disease. The American journal of pathology 2003, 163 (4), 1301-11.

5. Gao, B.; Bataller, R., Alcoholic liver disease: pathogenesis and new therapeutic targets. Gastroenterology 2011, 141 (5), 1572-85.

6. You, M.; Jogasuria, A.; Taylor, C.; Wu, J., Sirtuin 1 signaling and alcoholic fatty liver disease. Hepatobiliary surgery and nutrition 2015, 4 (2), 88-100.

7. Harris, T. E.; Finck, B. N., Dual function lipin proteins and glycerolipid metabolism. Trends in endocrinology and metabolism: TEM 2011, 22 (6), 226-33.

8. Everitt, H.; Hu, M.; Ajmo, J. M.; Rogers, C. Q.; Liang, X.; Zhang, R.; Yin, H.; Choi, A.; Bennett, E. S.; You, M., Ethanol administration exacerbates the abnormalities in hepatic lipid oxidation in genetically obese mice. American 
journal of physiology. Gastrointestinal and liver physiology 2013, 304 (1), G38-47.

9. Isabelle, M.; Moreel, X.; Gagne, J. P.; Rouleau, M.; Ethier, C.; Gagne, P.; Hendzel, M. J.; Poirier, G. G., Investigation of PARP-1, PARP-2, and PARG interactomes by affinity-purification mass spectrometry. Proteome science $\mathbf{2 0 1 0 ,}$ 8, 22 .

10. Kraus, W. L.; Hottiger, M. O., PARP-1 and gene regulation: progress and puzzles. Molecular aspects of medicine 2013, 34 (6), 1109-23.

11. Hans, C. P.; Zerfaoui, M.; Naura, A. S.; Troxclair, D.; Strong, J. P.; Matrougui, K.; Boulares, A. H., Thieno[2,3-c]isoquinolin-5-one, a potent poly(ADP-ribose) polymerase inhibitor, promotes atherosclerotic plaque regression in high-fat diet-fed apolipoprotein E-deficient mice: effects on inflammatory markers and lipid content. The Journal of pharmacology and experimental therapeutics 2009, 329 (1), 150-8.

12. Zakaria, E. M.; El-Maraghy, N. N.; Ahmed, A. F.; Ali, A. A.; El-Bassossy, H. M., PARP inhibition ameliorates nephropathy in an animal model of type 2 diabetes: focus on oxidative stress, inflammation, and fibrosis. Naunyn-Schmiedeberg's archives of pharmacology 2017, 10.1007/s00210-017-1360-9.

13. Fernando, H.; Wiktorowicz, J. E.; Soman, K. V.; Kaphalia, B. S.; Khan, M. F.; Shakeel Ansari, G. A., Liver proteomics in progressive alcoholic steatosis. Toxicology and applied pharmacology 2013, 266 (3), 470-80.

14. Sistigu, A.; Manic, G.; Obrist, F.; Vitale, I., Trial watch - inhibiting PARP 
enzymes for anticancer therapy. Molecular \& cellular oncology 2016, 3 (2), e1053594.

15. Islam, B. U.; Habib, S.; Ali, S. A.; Moinuddin; Ali, A., Role of Peroxynitrite-Induced Activation of Poly(ADP-Ribose) Polymerase (PARP) in Circulatory Shock and Related Pathological Conditions. Cardiovascular toxicology 2016, 10.1007/s12012-016-9394-7.

16. Vida, A.; Marton, J.; Miko, E.; Bai, P., Metabolic roles of poly(ADP-ribose) polymerases. Seminars in cell \& developmental biology 2016, 10.1016/j.semcdb.2016.12.009.

17. Huang, K.; Du, M.; Tan, X.; Yang, L.; Li, X.; Jiang, Y.; Wang, C.; Zhang, F.; Zhu, F.; Cheng, M.; Yang, Q.; Yu, L.; Wang, L.; Huang, D.; Huang, K., PARP1-mediated PPARalpha poly(ADP-ribosyl)ation suppresses fatty acid oxidation in non-alcoholic fatty liver disease. Journal of hepatology 2016, 10.1016/j.jhep.2016.11.020.

18. Gariani, K.; Ryu, D.; Menzies, K. J.; Yi, H. S.; Stein, S.; Zhang, H.; Perino, A.; Lemos, V.; Katsyuba, E.; Jha, P.; Vijgen, S.; Rubbia-Brandt, L.; Kim, Y. K.; Kim, J. T.; Kim, K. S.; Shong, M.; Schoonjans, K.; Auwerx, J., Inhibiting poly ADP-ribosylation increases fatty acid oxidation and protects against fatty liver disease. Journal of hepatology 2017, 66 (1), 132-141.

19. Rappou, E.; Jukarainen, S.; Rinnankoski-Tuikka, R.; Kaye, S.; Heinonen, S.; Hakkarainen, A.; Lundbom, J.; Lundbom, N.; Saunavaara, V.; Rissanen, A.; Virtanen, K. A.; Pirinen, E.; Pietilainen, K. H., Weight Loss Is Associated With 
Increased $\mathrm{NAD}(+) / \mathrm{SIRT} 1$ Expression But Reduced PARP Activity in White Adipose Tissue. The Journal of clinical endocrinology and metabolism 2016, 101 (3), 1263-73.

20. Shetty, P. K.; Galeffi, F.; Turner, D. A., Nicotinamide pre-treatment ameliorates $\mathrm{NAD}(\mathrm{H})$ hyperoxidation and improves neuronal function after severe hypoxia. Neurobiology of disease 2014, 62, 469-78.

21. Banasik, M.; Stedeford, T.; Strosznajder, R. P., Natural inhibitors of poly(ADP-ribose) polymerase-1. Molecular neurobiology 2012, 46 (1), 55-63.

22. Martire, S.; Mosca, L.; d'Erme, M., PARP-1 involvement in neurodegeneration: A focus on Alzheimer's and Parkinson's diseases. Mechanisms of ageing and development 2015, 146-148, 53-64.

23. Mouchiroud, L.; Houtkooper, R. H.; Auwerx, J., NAD $(+)$ metabolism: a therapeutic target for age-related metabolic disease. Critical reviews in biochemistry and molecular biology 2013, 48 (4), 397-408.

24. Yu, J. H.; Song, S. J.; Kim, A.; Choi, Y.; Seok, J. W.; Kim, H. J.; Lee, Y. J.; Lee, K. S.; Kim, J. W., Suppression of PPARgamma-mediated monoacylglycerol O-acyltransferase 1 expression ameliorates alcoholic hepatic steatosis. Scientific reports 2016, 6, 29352.

25. Mukhopadhyay, P.; Horvath, B.; Rajesh, M.; Varga, Z. V.; Gariani, K.; Ryu, D.; Cao, Z.; Holovac, E.; Park, O.; Zhou, Z.; Xu, M. J.; Wang, W.; Godlewski, G.; Paloczi, J.; Nemeth, B. T.; Persidsky, Y.; Liaudet, L.; Hasko, G.; Bai, P.; Boulares, A. H.; Auwerx, J.; Gao, B.; Pacher, P., PARP inhibition protects against alcoholic 
and non-alcoholic steatohepatitis. Journal of hepatology 2017, 66 (3), 589-600.

26. Pang, J.; Cui, J.; Gong, H.; Xi, C.; Zhang, T. M., Effect of NAD on PARP-mediated insulin sensitivity in oleic acid treated hepatocytes. Journal of cellular physiology 2015, 230 (7), 1607-13.

27. Kiss, B.; Szanto, M.; Szklenar, M.; Brunyanszki, A.; Marosvolgyi, T.; Sarosi, E.; Remenyik, E.; Gergely, P.; Virag, L.; Decsi, T.; Ruhl, R.; Bai, P., Poly(ADP) ribose polymerase-1 ablation alters eicosanoid and docosanoid signaling and metabolism in a murine model of contact hypersensitivity. Molecular medicine reports 2015, $11(4), 2861-7$.

28. Bai, P.; Csoka, B., New route for the activation of poly(ADP-ribose) polymerase-1: a passage that links poly(ADP-ribose) polymerase-1 to lipotoxicity? The Biochemical journal 2015, 469 (2), e9-11.

29. Canto, C.; Houtkooper, R. H.; Pirinen, E.; Youn, D. Y.; Oosterveer, M. H.; Cen, Y.; Fernandez-Marcos, P. J.; Yamamoto, H.; Andreux, P. A.; Cettour-Rose, P.; Gademann, K.; Rinsch, C.; Schoonjans, K.; Sauve, A. A.; Auwerx, J., The $\mathrm{NAD}(+)$ precursor nicotinamide riboside enhances oxidative metabolism and protects against high-fat diet-induced obesity. Cell metabolism 2012, 15 (6), 838-47. 